\title{
Stocks' Trading System Based on the Particle Swarm Optimization Algorithm
}

\author{
Jovita Nenortaite and Rimvydas Simutis \\ Vilnius University Kaunas Faculty of Humanities, \\ Muitines st. 8,3000 Kaunas, Lithuania \\ \{jovita.nenortaite, rimvydas.simutis\}@vukhf.lt
}

\begin{abstract}
One of the central problems in financial markets is to make the profitable stocks trading decisions using historical stocks' market data. This paper presents the decision-making method which is based on the application of neural networks (NN) and swarm intelligence technologies and is used to generate one-step ahead investment decisions. In brief, the analysis of historical stocks prices variations is made using "single layer" NN, and subsequently the Particle Swarm Optimization (PSO) algorithm is applied in order to select "global best" NN for the future investment decisions and to adapt the weights of other networks towards the weights of the best network. The experimental investigations were made considering different number of $\mathrm{NN}$, moving time intervals and commission fees. The experimental results presented in the paper show that the application of our proposed method lets to achieve better results than the average of the market.
\end{abstract}

\section{Introduction}

The continuing improvements of computer technologies, telecommunication services' grow make a big influence on globalization of stock markets and more efficient its information processing tools are required. The complexity and "noisiness" of stock markets cause difficulties in making real time analysis of it and forecasting its changes in the future. It was proved that having complex systems a collection of individuals often solves a problem better than an individual even an expert [1. Individuals acting within "a swarm" interact with each other in order to solve a global objective in a more efficient manner than a single individual could 8 .

The main objective of this paper is to develop the method based on artificial intelligence tools, which includes artificial NN, swarm intelligence technologies (particle swarm optimization), and apply it for the decisions-making in stocks' trading markets.

PSO algorithm is one of the swarm intelligence algorithms. It is a populationbased search algorithm based on the simulation of the social behavior among individuals (particles) "moving" through a multidimensional search space. Each particle of the swarm represents a candidate solution to the optimization of the problem. While each particle is "flown" through the multidimensional search 
space it is adjusting its position in search space according to its own experience and that of neighboring particles [3]. The PSO is closely related to evolutionary computation and artificial life (A-life) in general. The same as evolutionary programming it is highly dependent on stochastic processes. The optimizer which is used in the PSO algorithm, while making adjustment towards "local" and "global" best particles, is conceptually similar to the crossover operation used by genetic algorithms [8]. As well PSO includes fitness function, which measures the closeness of the corresponding solution to the optimum. The same function is included in the paradigms of evolutionary computation. The main difference of PSO concept from the evolutionary computing is that flying potential solutions through hyperspace are accelerating toward "better" solutions, while in evolutionary computation schemes operate directly on potential solutions which are represented as locations in hyperspace [7.

The problem of stock markets forecasting was analyzed by many researchers in the past. Considerable efforts have been put into investigation of stock markets changes and creating its forecasting systems. There are not so many examples of Swarm Intelligence applications for the solving of this problem. However, the published examples of swarm intelligence applications seem to be promising and give good results. In paper [10] there is proposed the forecasting methodology for the daily exchange rates of Japanese Yen against the US Dollar and of the US Dollar to the British Pound. The proposed forecasting methodology includes clustering technology, artificial NN and evolutionary computing. In contrast to this paper we focus on the formation of recommendations while making investment decision in stocks' markets. As well we are working with a large data set that lets us to propose more stable investment decision system. In paper [2] the authors are focusing on adapting PSO to dynamic environments. This paper is more focused on the modifications of PSO algorithm, while our goal is to introduce the investment decision-making method where PSO will be only one constituent of it.

The paper is organized as follows: Section 2 presents the problem and describes the proposed method; Section 3 is devoted for the implementation of PSO algorithms; In Section 3 the experimental results are discussed. The main conclusions of the work are presented in Section 4.

\section{Description of Proposed Method}

The analysis and forecast of stock market variations is stickler, because of its complexity and noisiness. There is not enough to use conventional techniques to conduct the stock markets predictions, as its changes are influenced by stochastic human factors, nonlinear, multivariable and temporal nature of stock price transitions. The use of artificial intelligence had made a big influence on the forecasting and investment decision-making technologies and it was proved that the efficient results can be obtained [4, [6]. In this paper we are proposing a decision making method, which is based on the study of historical data, the use of NN and PSO algorithm. 
In experimental investigations we are using 350 stocks, which are taken from the list of SP500 index. The data set represents stocks' price changes for 12 years (01/Oct/91-01/Oct/03). In our method we are using "single layer" NN, with the designation "single layer" referring to the output layer of computation nodes (neurons). The signals (data set) are passed to the cell body. Once they reach it, they are combined additively. The net result is a linear combination of each of the weighted input vectors. The NN' weights are initialized randomly at the beginning of the procedure. The random numbers are kept small, and symmetrical about zero. In the investigations there are considered stocks' prices changes for 5 days. The changes of 5 days were taken making an assumption that such stocks' prices changes have the highest correlation with the next day stocks price change [11]. For example, if stocks' price changes of five days are negative, there is a high possibility that the price of these stocks will increase on the next day and vise versa. Further, for each day, and each stock the recommendations are calculated using different number of "single layer" NN and taking into account prices changes of different number of days. The net result is passed to the hyperbolic tangent function and the recommendations for the stocks' trading are calculated. The recommendations represent the relative rank of investment attraction to each stock in the interval $[-1,1]$. The values $-1,0$ and +1 represent recommendations: sell, hold and buy respectively. After the recommendations are calculated, all the stocks are sorted in the descending order. Having sorted stocks we are taking into account 3 stocks (for each day) that have the highest recommendations. Further, the observation of these stocks price changes behavior on the next day is made considering different length moving time intervals. This observation lets us to see how good results could we get making the decisions according to the price changes of the stocks with the highest recommendations. The next step in our method is the use of PSO algorithm and the selection of "global best" particles, which will be used for the calculations of expected profit. The more detailed explanation of PSO algorithm is presented in the next section.

\section{Application of Particle Swarm Optimization Algorithm}

In this paper our objective is to apply the PSO algorithm, which is based on the search of "global best" particle. Here particles are represented as "single layer" NN. So called "global best" particle is chosen for every day taking into account the chosen moving time interval, it means that every day we are comparing the performance (fitness function) of NN and the network with the highest performance is chosen for further experimental investigations. The adaptation of the other particles weights is made towards the weights of "global best" particle. Such adaptation of weights and training of NN let us to move towards the best solution as all the time the trading decision are made using NN that have shown the best performance. Knowing the day and NN, which performance was the best on that day, we are selecting 3 stocks with the highest recommendations. Further the calculation of the prices' change mean of 3 stocks with the highest 
recommendations is made. We believe that the investigation of stocks with the highest recommendations will let to achieve the best gain on the next trading day. More detailed explanations of experimental investigations are presented in the next Section.

\section{Experimental Investigations}

The realization of our proposed method was made using MATLAB software package. All our made experimental investigations could be described in several functional steps:

1. Stocks' prices relative changes $(\%)$ for the time period $01 /$ Oct $/ 91-01 /$ Oct/03 were calculated.

2. The recommendations for the purchase of the stock were formed using "single layer" NN.

3. All the recommendations were sorted and for every day three stocks with the highest recommendations were chosen.

4. The price changes of chosen stocks on the next day were explored.

5. There were calculated expected day profits, taking into account the price changes of chosen stocks and the profit estimations was made selecting different moving time intervals. This profit was calculated as a sum of stock prices changes $(\%)$.

6. The PSO algorithm was applied and the profit estimation based on the search of "global best" particles was made.

7. In parallel there was estimated the profit, making an assumption that the investments were made into SP500 index.

8. The experiments were run taking into account different number of $\mathrm{NN}$ and different size moving time intervals in order to find the situations when the best and the most stable results could be achieved.

\subsection{Selection of Moving Time Interval and Number of Neural Networks}

The first part of experimental investigations was focused on the choice of optimal number of NN and on the choice of the size of moving time interval. The experiments were run taking into account different size moving time intervals and different number of NN. The number of explored stocks and days was the same for all the cases. In these experimental investigations the commission fee for selling and buying stocks was not considered. The obtained results let us to make several conclusions:

- The bigger number of NN, with different initial weights, let us to achieve more stable results (see Fig. 1] and 2).

- The bigger moving time intervals let to avoid unnecessary variations and to achieve better results (see Fig. 3). 


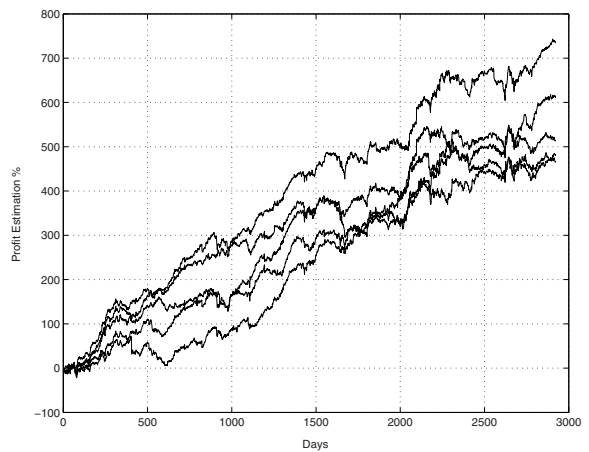

Fig. 1. Profit estimation (5 NN, moving time interval 100 days)

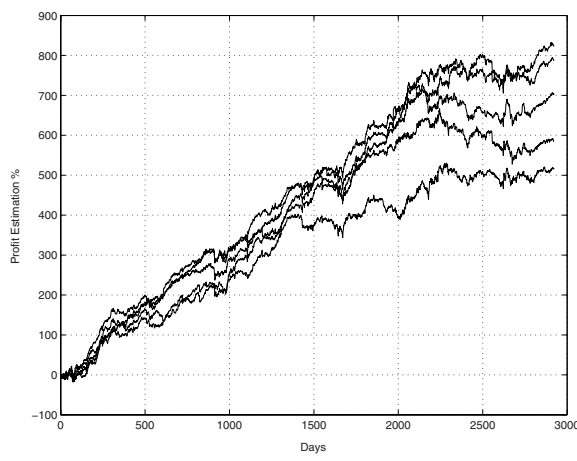

Fig. 2. Profit estimation (30 NN, moving time interval 100 days)

- The experimental investigations showed that the best results are achieved while taking $30 \mathrm{NN}$ and the moving time interval of 100 days.

From the Fig. 1 and 2 it can be seen that the swing of the results is much smaller when there were taken $30 \mathrm{NN}$. As well the experimental investigations showed that the results are influenced by the choice of moving time interval size. Fig. 3 shows how the profit (\% per day) is correlated with the different size moving time intervals while exploring $30 \mathrm{NN}$. Here numbers $1,2, \ldots, 10$ represent time intervals $10,20, \ldots, 100$ respectively.

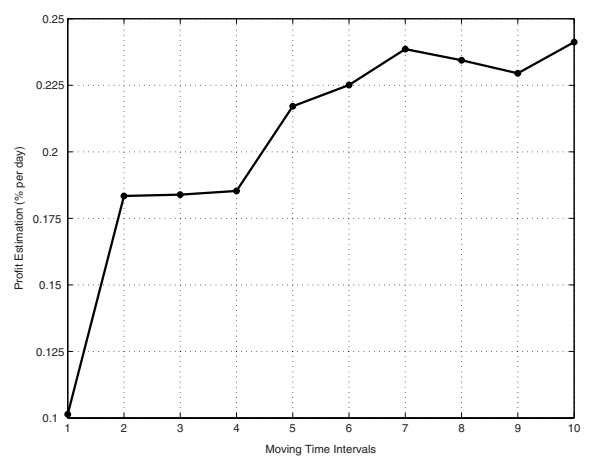

Fig. 3. Correlation between profit and moving time intervals

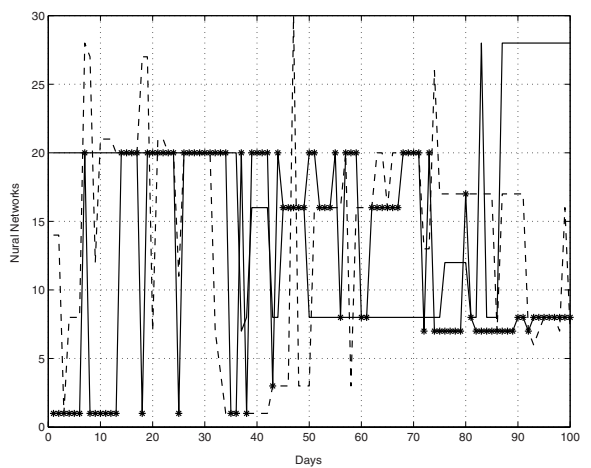

Fig. 4. The swing of NN depending from the moving time interval

As it can be seen from the Fig. 3, the profit is growing while increasing moving time intervals. The variations of the profit become more stable while having moving time intervals from 70 to 100 days (see Fig. 44). The Fig. 4 shows that having moving time interval of 100 days (solid line) the swing between NN (having $30 \mathrm{NN}$ ) is much smaller than having the moving time interval of 10 
days (dashed line) or 50 days (bold line). Here the swing of $\mathrm{NN}$ is meant to be the choice of different "global best" NN depending from the time period. For example: having moving time interval of 100 days (solid line) we can see that starting from the 1st day and until the 38th day the "global best" NN is 20th, while during the same time period, having moving time intervals of 10 and 50 days the "global best" NN are changing almost every 3rd or 5th days. The results could be even more increased taking into account more NN and bigger moving time interval. In this paper there were not made such experimental investigations because of the limited computer capacity.

The comparison of profit estimation results using our proposed method (having $30 \mathrm{NN}$ and moving time interval of 100 days) and results achieved while making investments into SP500 index are presented in Fig. 5]

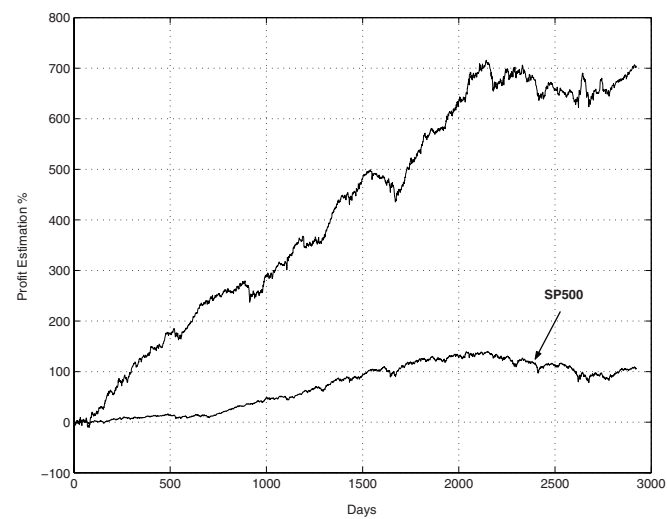

Fig. 5. Comparison of the results

As it can be seen from the Fig. 5 the application of PSO algorithm gives very good results. The profit accumulation results are 5-7 times better than the results achieved making investments into SP500 index. The obtained results confirm that using our method and not paying any commission fee we are able to beat the market. The good results are achieved as every day, while making the desicion, there is made an adaptation of particles' weights to the weights of "global best" and this lets to position all the particles towards the fitness of best particle.

\subsection{The Estimation of Profit Considering Account Commission Fee}

In the second part of experimental investigations we were focusing on the estimation of the profit considering commission fee. In the first part of experimental investigations we have got results showing, that having $30 \mathrm{NN}$, moving time interval of 100 days and not considering commission fee we are able to get 0.2344 
$\%$ average incomes per day. The analysis of the commission fees of different ebrokers showed that commission fee in real trading process is usually between $0.15 \%-0.3 \%$. For example, such transition fees are provided by the company of Interactive Brokers [5]. Having bigger selling and buying volumes this fee could be even smaller - $0.1 \%$. Based on that for further investigations we are considering the commission fee which is equal $0.15 \%$. We are making an assumption that on the first trading day we are investing $1000 \$$ into the market. Each day we are paying $0.15 \%$ of commission fee for buying new stocks. The value got on the last investigated trading day is considered as the profit. For the trading of SP500 index the commission fee is not considered. The experimental investigations showed that having the commission fee of $0.15 \%$ we are able to earn more than the average of the market (see Fig. 6).

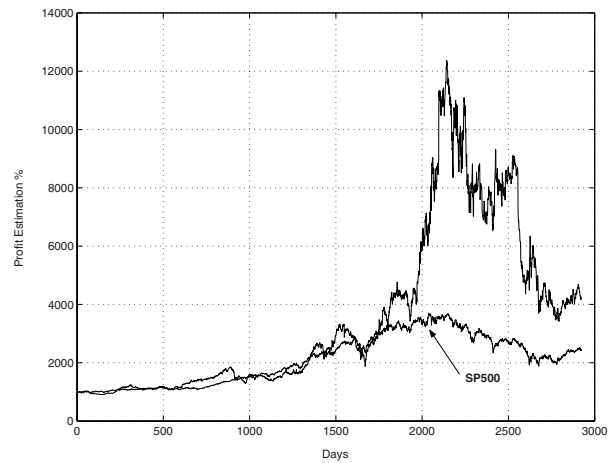

Fig. 6. Profit estimation considering commission fee of $0.15 \%$

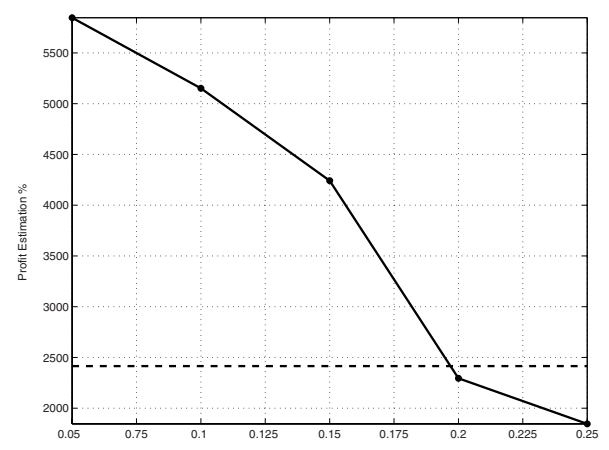

Fig. 7. Profit dependence from the commission fee

The situation is changing while the commission fee is increasing (see Fig. 17). While having the commission fee of $0.2 \%$ we are already slightly loosing compare to the profit that has been got from the investment into SP500 index. The experimental investigations show, that using our proposed method are able to win only big funds that have a possibility to pay small $(0.15 \%)$ commission fees for buying and selling stocks. The experimental investigations have showed that the results, achieved while applying our proposed method, are much better as it was got using NN for the investigation of similar time series and its future changes forecasting [9], 12]. However, the proposed method still has to be improved in order to achieve better results while considering bigger commission fees.

\section{Conclusions and Future Work}

In this paper we proposed the investment method based on PSO algorithm. The method was applied in order to make one-step ahead profit estimation conside- 
ring historical data of stocks' price changes. The experimental investigations let as to make the following conclusions:

1. The bigger number of NN and longer moving time interval let us to achieve better and more stable results.

2. The profit accumulation results, while not considering any commission fees, were 5-7 times better than the results achieved making investments into SP500 index.

3. Taking into account the commission fee of $0.15 \%$ the proposed method gives better results than the average of the market.

4. The bigger commission fees (around $0.2 \%$ ) reduce the results and the investment into SP500 index becomes more reasonable.

5. The propose method could be useful for big financial funds that are able to pay small commissions fees for buying and selling stocks.

In the future we intend to make more detailed analysis of our proposed method.

\section{References}

1. Bartholdson K., Mauboussin J.M.: Thoughts on Organizing for Investing Success. Credit Suisse First Boston Equity Research (2002)

2. Carlisle A., Dozier G.: Adapting Particle Swarm Optimization to Dynamic Environments. 2000 ICAI Proceedings, Las Vegas (2000) 429-434

3. Engelbrecht A.D.: Computational Intelligence (An Introduction). John Wiley \& Sons, London (2002)

4. Hellstrom T.: Optimizing the Sharpe Ration for a Rank Based Trading System. Lecture Notes in Artificial Intelligence, LNA 2258. Springer-Verlag, New York (2001)

5. Interactive Brokers: http://www.interactivebrokers.com. Current as of February 9th, 2004.

6. Kaastra I., Milton B.: Designing a Neural Network for Forecasting Financial and Economic Time Series. Neurocomputing (1996)

7. Kennedy J., Spears W.M.: Matching Algorithms to Problems: An Experimental Test of the Particle Swarm and Some Genetic Algorithms on the Multimodal Problem Generator. http://www.aic.nrl.navy.mil/\%7Espears/papers/wcci98.pdf. Current as of December 15th, 2003.

8. Khalil A.S.: An Investigation into Optimization Strategies of Genetic Algorithms and Swarm Intelligence. Artificial Life (2001)

9. Lowe D., Webb A.R.: Time Series Prediction by Adaptive Networks: A Dynamical Systems Perspective. IEEE Computer Society Press (1991)

10. Pavlidis N.G., Tasoulis D.,Vrahatis M.N.: Financial Forecasting Through Unsupervised Clustering and Evolutionary Trained Neural Networks. 2003 Congress on Evolutionary Computation, Canberra Australia (2003)

11. Simutis R.: Stock Trading Systems Based on Stock's Price Ranks (in Lithuanian). Ekonomika (2003)

12. White H.: Economic Prediction Using Neural Networks: The Case of IBM Daily Stock Returns. IEEE International Conference on Neural Networks, San Diego (1988) 